\title{
An Adaptive Sliding Mode Observer for A Complex Network of Dynamical Systems
}

\author{
Prathyush P. Menon* Christopher Edwards** \\ * Centre for Systems, Dynamics and Control, Mathematical Research \\ Institute, University of Exeter, Exeter, EX4 4JN, U.K. (e-mail: \\ P.M.Prathyush@exeter.ac.uk) \\ ** Control Group, Department of Engineering, University of Leicester, \\ Leicester, LE1 7RH, U.K. (e-mail: ce14@le.ac.uk)
}

\begin{abstract}
A novel approach for reconstructing the entire state information of all the individual nodes of a complex network dynamical system, at a supervisory level, is proposed in this paper. The nodes of the network are assumed to belong to a class of systems with a known linear part and unknown but bounded nonlinearities or uncertainties in certain channels of the system. Adaptive sliding mode observers are designed for the purpose of enhancing autonomy in the network of dynamical systems. The synthesis of the sliding mode observer, formulated as a system of LMIs, depends entirely on the dynamics of an individual node of the network. The efficacy of the proposed methodology is demonstrated by considering a network of Chua oscillators.
\end{abstract}

Keywords: Sliding Mode, Observers, Adaptive, Complex Networks

\section{INTRODUCTION}

The control and analysis of networks consisting of a large number of dynamical systems, has gained significant research attention in recent years (Mesbahi and Egerstedt [2010], Ji and Chen [2007], Fax and Murray [2004], Wu [2007]). Centralised monitoring of the system behaviour is important, and in practical engineering systems, safety and reliability is critical and an important issue to be addressed. A specific representation of the general scale-free dynamical network model, in a modified form as reported in (Menon and Edwards [2008]), consistent with the one in (Wu [2007]), will be utilized in this paper. The motivation for the work reported in the sequel is the need to have an increased level of autonomy in the case of 'systems of systems' i.e. a group of dynamical systems operated over a network, and controlled to perform synchronously or to attain a common objective. Systems operated in such environments are prevalent in many areas of research - for example mobile robots, cooperating UAV team operations, formation flying of UAV's and satellites, vehicle platoons, and distributed state estimation applications such as localisation: for details, see for example (Mesbahi and Egerstedt [2010]).

Sliding mode observers, which are inherently robust nonlinear observers, have been developed over a number of years (Drakunov and Utkin [1995], Koshkouei and Zinober [2000], Floquet and Barbot [2004], Edwards and Spurgeon [1994, 1998], Bejarano et al. [2007], Fridman et al. [2007a], Floquet and Barbot [2006], Chen and Saif [2007], Fridman et al. [2007b]). Often, the structure of the problem is exploited for designing appropriate novel observer gains for different systems. However, the underlying principle is to drive the state estimation error to the sliding plane in finite time and thereby achieve asymptotic observers for the network of subsystems which are applicable to nonlinear and time varying systems. In (Azemi and Yaz [2000]) an adaptive sliding mode observer has been designed for synchronisation of coupled nonlinear systems (in a masterslave architecture). In (Raoufi and Zinober [2006]) a sliding mode observer has been applied to crypto-systems - an- other relevant area of synchronisation of coupled masterslave nonlinear systems. Adaptive sliding mode control has also been applied in (Yan et al. [2006]) for synchronisation of general master slave systems. Note all these applications follow a master-slave framework. Recently a second order sliding mode observer has been designed specifically for online monitoring and fault detection in satellite formations, in the presence of uncertainty (Wu and Saif [2007]). Patton and co-workers have recently proposed a generic framework for FDI in networked systems (Patton et al. [2007], Kambhampati et al. [2007]) based on unknown input observers. In (Noijen et al. [2005]), an observercontroller combination is discussed for a single unicycle mobile robot. Mariottini et al. [2005] consider a similar problem, providing a sufficient condition for nonlinear observability and an associated Extended Kalman Filter (EKF) scheme for the localization problem.

The synthesis of a network monitoring observer, for a class of dynamical systems operating over an undirected network is addressed in this paper. The individual node level dynamics are represented as a combination of a linear system and a class of unknown bounded nonlinear/uncertain parts. The underlying principle is to estimate the entire state of the network at a supervisory node level from only measurement signals using an adaptive sliding mode observer. In this paper, the bounds of the nonlinearities/uncertainties/disturbances present in certain distribution channels are unknown, however they are assumed to be bounded. The adaptive gains are used in the sliding mode observer to estimate the states robustly. A systematic approach for state reconstruction of the network is developed based on existing sliding mode observer ideas.

\section{SYSTEM DESCRIPTION}

In this paper, a scale free network of $N$ nearly identical interconnected dynamical systems, indexed as $1,2, \ldots, N$, is considered. The interconnections among the dynamical systems are assumed to be of bidirectional nature, and viewed as an undirected graph where node and edge rep- 
resent $n$ dimensional dynamical system and the interconnections respectively. Specifically:

$$
\begin{aligned}
& \dot{x}_{i}=A x_{i}+D \xi_{i}\left(x_{i}, u_{i}\right)+B u_{i}-\alpha(t) \sum_{j=1}^{N} \mathcal{L}_{i j} \Gamma x_{j} \\
& y_{i}=C x_{i}
\end{aligned}
$$

where $x_{i} \in \mathbb{R}^{n}, u_{i} \in \mathbb{R}^{m}$ and $y_{i} \in \mathbb{R}^{p}$ represent the state, the control input and the measured output vector of the $i^{t h}$ node respectively. The term $\xi_{i}$ represents an unknown bounded uncertainty (or unknown nonlinearity) or a disturbance present in the dynamics, but assumed it is to satisfy a bound $\left\|\xi_{i}\left(x_{i}, u_{i}\right)\right\| \leq \beta_{0}$. The constant term $\beta_{0}$ is assumed to be unknown but finite.

Assumption 1. The triple $(A, D, C)$ is assumed to be a minimal realization, and consists of constant matrices $A \in \mathbb{R}^{n \times n}, D \in \mathbb{R}^{n \times q}$ and $C \in \mathbb{R}^{p \times n}$, where $C$ and $D$ are full row and column rank respectively. Moreover, it is assumed that $p \geq q$.

Assumption 2. The system $(A, D, C)$ is minimum phase.

Assumption 3. At each node level, it is assumed that the distribution matrices satisfy the condition, $\operatorname{rank}(C D)=q$.

The matrix $\Gamma=\tau_{i j} \in \mathbb{R}^{n \times n}$ is the local coupling configuration among the states of the nodes, whose entries are either 0 or 1 and represents the existence or non-existence of coupling in the respective channels in the network. The Laplacian of $\mathcal{G}$, written as $\mathcal{L} \in \mathbb{R}^{N \times N}$, represents the interconnections of the network being considered. Whenever node $i$ interacts with node $j$, i.e the existence of an edge between nodes $i$ and $j$, then $\mathcal{L}_{i j}=\mathcal{L}_{j i}=-1$ and otherwise $\mathcal{L}_{i j}=\mathcal{L}_{j i}=0$. The diagonal terms are given by $\mathcal{L}_{i i}=k_{i}$, for $i=1, \ldots, N$ where $k_{i}$ is the degree of node $i$, and represents the total number of connections incident at the $i^{\text {th }}$ node. The smallest eigenvalue of $\mathcal{L}$ is zero and the corresponding eigenvector is given by $\mathbf{1}$. Furthermore, the graph Laplacian is a symmetric positive semi-definite matrix (Royle and Godsil [2001]). The Laplacian matrix, $\mathcal{L}$, has a "row sum equal to zero" property and this will be exploited in this paper. The scalar $\alpha(t) \in \mathbb{R}^{+}$for all $t \in \mathbb{R}^{+}$is a known time varying coupling strength. It is assumed that $\alpha(t)$ has a known upper bound $\hat{\alpha}=\sup _{t \in \mathbb{R}^{+}} \alpha(t)$.

The problem addressed in this paper is to design an adaptive sliding mode observer at a supervisory level to robustly reconstruct the entire state of the network $x=\operatorname{Col}\left(x_{1}, \ldots, x_{N}\right)$ based only on information about the measured outputs $y=\operatorname{Col}\left(y_{1}, \ldots, y_{N}\right)$ and the control inputs $u=\operatorname{Col}\left(u_{1}, \ldots, u_{N}\right)$.

\section{SLIDING MODE NETWORK OBSERVER}

Under Assumptions 2 and 3, there exists a nonsingular linear orthogonal transformation $x_{i} \mapsto T_{0} x_{i}$, for $i=1, \ldots, N$, transforming the original co-ordinates to ones in which the system triple $(A, D, C)$ has the following canonical structure (see Section 5.3 in Edwards and Spurgeon [1998])

$$
A=\left[\begin{array}{ll}
A_{11} & A_{12} \\
A_{21} & A_{22}
\end{array}\right] \quad D=\left[\begin{array}{c}
0 \\
D_{0}
\end{array}\right] \quad C=\left[\begin{array}{ll}
0 & C_{2}
\end{array}\right]
$$

where $A_{11} \in \mathbb{R}^{(n-p) \times(n-p)}, D_{0} \in \mathbb{R}^{q \times q}$ is non-singular, and $C_{2} \in \mathbb{R}^{p \times p}$ is orthogonal. Define $A_{211}$ as the top $p-q$ rows of $A_{21}$. By construction, the pair $\left(A_{11}, A_{211}\right)$ is detectable and the unobservable modes of $\left(A_{11}, A_{211}\right)$ are the invariant zeros of $(A, D, C)$ Edwards et al. [2000]. For convenience, define $D_{2}=\operatorname{Col}\left[\begin{array}{ll}0 & D_{0}\end{array}\right] \in \mathbb{R}^{p \times q}$ as the bottom $p$ rows of $D$. This coordinate system will be used for the observer synthesis.

In the coordinate system of (3), write

$$
\Gamma=\left[\begin{array}{ll}
\Gamma_{11} & \Gamma_{12} \\
\Gamma_{21} & \Gamma_{22}
\end{array}\right]
$$

where $\Gamma_{22} \in \mathbb{R}^{p \times p}$. Note that the initial structure of $\Gamma$ is typically lost in achieving the canonical form in (3).

The state estimate of the $i^{t h}$ node is $z_{i} \in \mathbb{R}^{n}$. Consistent with the 4-block partition of $(A, D, C)$ in (3), define $z_{1 i} \in$ $\mathbb{R}^{n-p}$ and $z_{2 i} \in \mathbb{R}^{p}$ such that $\mathcal{C}$ ol $\left(z_{1 i}, z_{2 i}\right)=z_{i}$.

Consider the supervisory observer dynamical system described by

$$
\dot{z}_{i}=A z_{i}+B u_{i}-\alpha(t) \sum_{j=1}^{N} \mathcal{L}_{i j} \Gamma z_{j}-G_{l_{i}} e_{y_{i}}-G_{n_{i}} \nu_{i}
$$

for $i=1, \ldots, N$ where $e_{y_{i}}=C\left(x_{i}-z_{i}\right)$ and the global output error estimate is given by $e_{y}=\operatorname{Col}\left(e_{y_{1}}, \ldots e_{y_{N}}\right)$. Consistent with the partition in (3), partition $z_{i}=\mathcal{C}$ ol $\left(z_{1 i}, z_{2 i}\right)$ such that $z_{1 i} \in \mathbb{R}^{n-p}$ and $z_{2 i} \in \mathbb{R}^{p}$.

The discontinuous injection term, $\nu_{i}$, for $i=1, \ldots, N$, is given by:

$$
\nu_{i}=-\rho(t) \frac{e_{y_{i}}}{\left\|e_{y_{i}}\right\|}, \quad \text { if } e_{y_{i}} \neq 0
$$

The scalar modulation function $\rho: \mathbb{R}_{+} \mapsto \mathbb{R}_{+}$, which satisfies an adaptive law, will be described formally later.

The idea is to design the gain matrices $G_{l_{i}} \in \mathbb{R}^{n \times p}$ and $G_{n_{i}} \in \mathbb{R}^{n \times p}$ to ensure the state estimation error is driven to an a-priori defined sliding surface in finite time, with a defined performance level. A suitable choice for the matrix $G_{n_{i}}$ in the co-ordinate system in (3) is $G_{n_{i}}=\operatorname{Col}\left(L C_{2}^{\mathrm{T}}, C_{2}^{\mathrm{T}}\right)$, where $L=\left[\begin{array}{ll}L^{o} & 0\end{array}\right]$ with $L^{o} \in$ $\mathbb{R}^{(n-p) \times(p-q)}\left(\right.$ Edwards et al. [2000]). The matrices $L^{o}$ and $G_{l_{i}}$ are to be determined. A precise choice for $G_{l_{i}}$ will be developed in the sequel.

Define the error in the state estimate of the $i^{\text {th }}$ node as $e_{i}:=x_{i}-z_{i}$. It can be easily verified that the error dynamics satisfy

$\dot{e}_{i}=A e_{i}+D \xi_{i}\left(x_{i}, u_{i}\right)-\alpha(t) \sum_{j=1}^{N} \mathcal{L}_{i j} \Gamma e_{j}+G_{l_{i}} e_{y_{i}}+G_{n_{i}} \nu_{i}$

for $i=1, \ldots, N$. In the coordinates of (3), define $e_{1_{i}} \in$ $\mathbb{R}^{n-p}$ and $e_{2_{i}} \in \mathbb{R}^{p}$ such that $\operatorname{Col}\left(e_{1_{i}}, e_{2_{i}}\right)=e_{i}$. Note that $e_{2_{i}}$ is 'equivalent' to the output error estimate $e_{y_{i}}$ in the sense that $e_{y_{i}}=C_{2} e_{2}$ where $\operatorname{det}\left(C_{2}\right) \neq 0$ and in fact $C_{2}^{\mathrm{T}} C_{2}=C_{2} C_{2}^{\mathrm{T}}=I$. For system (7), the objective is to induce a finite time sliding motion on the surface

$$
\mathcal{S}=\bigcap_{i=1 \ldots N}\left(\mathcal{S}_{i}=\left\{\left(e_{1_{i}}, e_{2_{i}}\right) \mid e_{2_{i}}=0\right\}\right)
$$

\subsection{Stability of reduced order sliding motion}

Suppose a sliding motion is attained on the surface $\mathcal{S}$ in finite time. Subsequently, the reduced order sliding motion associated with the error dynamics $e_{i}$, when $e_{2_{i}}=\dot{e}_{2_{i}}=0$ is: 


$$
\begin{aligned}
{\left[\begin{array}{c}
\dot{e}_{1_{i}} \\
0
\end{array}\right]=} & {\left[\begin{array}{l}
A_{11} \\
A_{21}
\end{array}\right] e_{1_{i}}+\left[\begin{array}{c}
0 \\
D_{2}
\end{array}\right] \xi_{i}\left(x_{i}, u_{i}\right) } \\
& -\alpha(t) \sum_{j=1}^{N} \mathcal{L}_{i j}\left[\begin{array}{c}
\Gamma_{11} \\
\Gamma_{21}
\end{array}\right] e_{1_{j}}+\left[\begin{array}{c}
L C_{2}^{\mathrm{T}} \\
C_{2}^{\mathrm{T}}
\end{array}\right] \nu_{e q, i}
\end{aligned}
$$

for $i=1, \ldots, N$, where $\nu_{e q, i}$ is the so-called equivalent injection necessary to maintain sliding and represents the 'average' switched signal (Utkin [1992], Edwards and Spurgeon [1998]).

When sliding occurs on $\mathcal{S}$ defined in (8), the so-called equivalent injection, $\nu_{e q, i}$ necessary to maintain sliding, satisfies (Edwards and Spurgeon [1998], Utkin [1992])

$C_{2}^{\mathrm{T}} \nu_{e q, i}=-A_{21} e_{1_{i}}-D_{2} \xi_{i}\left(x_{i}, u_{i}\right)+\alpha(t) \sum_{j=1}^{N} \mathcal{L}_{i j} \Gamma_{21} e_{1_{j}}(10)$

Substituting for $C_{2}^{\mathrm{T}} \nu_{e q, i}$ from (10) in the first $n-p$ channels of the reduced order dynamics in (9) yields

$\dot{e}_{1_{i}}=\underbrace{\left(A_{11}-L A_{21}\right)}_{\tilde{A}_{11}} e_{1_{i}}-\alpha(t) \sum_{j=1}^{N} \mathcal{L}_{i j} \underbrace{\left(\Gamma_{11}-L \Gamma_{21}\right)}_{\tilde{\Gamma}_{11}} e_{1_{j}}$

for $i=1, \ldots, N$.

Since identical dynamical systems are considered, a Kronecker product representation can be used to represent the network level dynamics. Hence, the reduced order sliding dynamics (11) at a network level can be written as

$$
\dot{e}_{1}=\left(I_{N} \otimes\left(A_{11}-L A_{21}\right)-\alpha(t)\left(\mathcal{L} \otimes \tilde{\Gamma}_{11}\right)\right) e_{1}
$$

where $e_{1}:=\mathcal{C} \operatorname{ol}\left(e_{1_{1}}, e_{1_{2}}, \ldots e_{1_{N}}\right)$. Since the graph Laplacian matrix $\mathcal{L}$ is symmetric, by spectral decomposition

$$
\mathcal{L}=V \Lambda V^{\mathrm{T}}
$$

where $\Lambda=\operatorname{Diag}\left(\lambda_{1}, \ldots, \lambda_{N}\right)$ are the real nonnegative eigenvalues (Royle and Godsil [2001]), and $V$ is an orthogonal matrix composed of the eigenvectors. As in (Menon and Edwards [2008]), consider a change of coordinates $e_{1} \mapsto T_{v} e_{1}=\eta$ where

$$
T_{v}:=\left(V^{\mathrm{T}} \otimes I_{(n-p)}\right)
$$

Because $V^{\mathrm{T}} V=I_{N}, T_{v}$ in (14) is an orthogonal transformation. From the properties of Kronecker products, $T_{v}\left(I_{N} \otimes \tilde{A}_{11}\right) T_{v}^{\mathrm{T}}=\left(I_{N} \otimes \tilde{A}_{11}\right)$ and

$$
T_{v}\left(\mathcal{L} \otimes \tilde{\Gamma}_{11}\right) T_{v}^{\mathrm{T}}=\left(V^{\mathrm{T}} \mathcal{L} V\right) \otimes \tilde{\Gamma}_{11}=\Lambda \otimes \tilde{\Gamma}_{11}
$$

Applying the transformation in (14) to (12), and using the results mentioned above, it follows

$$
\dot{\eta}=\left(I_{N} \otimes\left(A_{11}-L A_{21}\right)-\alpha(t)\left(\Lambda \otimes \tilde{\Gamma}_{11}\right)\right) \eta
$$

Note that $\Lambda$ is diagonal and hence (15) can be written in a node level representation of the form

$$
\dot{\eta}_{i}=\underbrace{\left(A_{11}-L A_{21}-\alpha(t) \lambda_{i} \tilde{\Gamma}_{11}\right)}_{\tilde{A}_{i}(t)} \eta_{i}
$$

for $\eta_{i}, i=1 \ldots N$ where $\eta=\operatorname{Col}\left(\eta_{1}, \ldots \eta_{N}\right)$. Since $\alpha(t)$ is unknown but defined to be a positive bounded scalar and $\lambda_{i} \geq 0$ for $i=1, \ldots, N$, the terms $\alpha(t) \lambda_{i} \geq 0$ for $i=1, \ldots, N$.

Consider a candidate quadratic Lyapunov function of the form, $V_{i}\left(\eta_{i}\right)=\eta_{i}^{\mathrm{T}} P_{1} \eta_{i}$, where $P_{1} \in \mathbb{R}^{(n-p) \times(n-p)}$ is a symmetric positive definite matrix, to analyze the stability of reduced order sliding motion in (16). The time derivative of $V_{i}\left(\eta_{i}\right)$ along the trajectories of the system (16) is given by

$$
\begin{aligned}
\left.\dot{V}_{i}\left(\eta_{i}\right)\right|_{(16)}= & \eta_{i}^{\mathrm{T}}\left(P_{1} \tilde{A}_{i}(t)+\tilde{A}_{i}(t)^{\mathrm{T}} P_{1}\right) \eta_{i} \\
= & \eta_{i}^{\mathrm{T}}\left(P_{1}\left(A_{11}-L A_{21}\right)+\left(A_{11}-L A_{21}\right)^{\mathrm{T}} P_{1}\right) \eta_{i} \\
& -\alpha(t) \lambda_{i} \eta_{i}^{\mathrm{T}}\left(P_{1} \tilde{\Gamma}_{11}+\tilde{\Gamma}_{11}^{\mathrm{T}} P_{1}\right) \eta_{i}
\end{aligned}
$$

Since $\alpha(t) \lambda_{i} \geq 0$, provided the term $\left(P_{1} \tilde{\Gamma}_{11}+\tilde{\Gamma}_{11}^{\mathrm{T}} P_{1}\right)$ is guaranteed to be positive semi-definite, then the timevarying expression $-\alpha(t) \lambda_{i} \eta_{i}^{\mathrm{T}}\left(P_{1} \tilde{\Gamma}_{11}+\tilde{\Gamma}_{11}^{\mathrm{T}} P_{1}\right) \eta_{i}$ in (17) is negative semi-definite for all $\eta_{i} \neq 0$. Hence, a sufficient condition to ensure the negative definiteness of $\dot{V}_{i}\left(\eta_{i}\right)$ in (17), for all $\eta_{i} \neq 0$ and for all $t \in \mathbb{R}^{+}$, is to synthesise a s.p.d matrix $P_{1} \in \mathbb{R}^{(n-p) \times(n-p)}$ and $L \in \mathbb{R}^{(n-p) \times p}$ such that

$$
\begin{array}{r}
-P_{1}\left(\Gamma_{11}-L \Gamma_{21}\right)-\left(\Gamma_{11}-L \Gamma_{21}\right)^{\mathrm{T}} P_{1} \leq 0 \\
P_{1}\left(A_{11}-L A_{21}\right)+\left(A_{11}-L A_{21}\right)^{\mathrm{T}} P_{1}+\tilde{q} I_{n-p} \leq 0
\end{array}
$$

where $\tilde{q} \in \mathbb{R}^{+}$is a design scalar fixed a-priori to ensure a certain level of quadratic performance associated with the reduced order sliding motion dynamics in (12). The inequalities in (18)-(19) can be formulated as a standard convex optimization problem via a system of LMI's in the matrix variables $M, P_{1}$, by making use of the change of variable $M:=P_{1} L \in \mathbb{R}^{(n-p) \times p}$. It can be seen from (18) and (19) that the LMI's to be solved are independent of the number of nodes in the network $N$ and depend on $(n-p) \times(n-p)+(n-p) \times(p-q)$ decision variables (since $M:=\left[\begin{array}{ll}M_{1} & 0\end{array}\right]$ where $\left.M_{1} \in \mathbb{R}^{(n-p) \times(p-q)}\right)$.

\subsection{Quadratic stability}

Once the matrix $L$ has been synthesized, define an invertible transformation $e_{i} \mapsto T_{L} e_{i}$, for $i=1, \ldots, N$ where

$$
T_{L}:=\left[\begin{array}{cc}
I_{n-p} & -L \\
0 & I_{p}
\end{array}\right]
$$

and $L:=\left[\begin{array}{ll}L^{o} & 0\end{array}\right] \in \mathbb{R}^{(n-p) \times p}$ with $L^{o} \in \mathbb{R}^{(n-p) \times(p-q)}$ (Edwards et al. [2000]). Consistent with (3), partition the error state vector in the transformed coordinates of (20) as $\operatorname{Col}\left(\tilde{e}_{1_{i}}, e_{2_{i}}\right)=T_{L} e_{i}$, for $i=1, \ldots, N$ where $\tilde{e}_{1_{i}}:=$ $e_{1_{i}}-L e_{2_{i}}$. Consistent with the partitioned form, define the gains associated with the linear injection as $G_{l_{i}}=$ $\operatorname{Col}\left(G_{1_{i}}, G_{2_{i}}\right)$, for $i=1, \ldots, N$ where $G_{1_{i}} \in \mathbb{R}^{(n-p) \times p}$. In the coordinates of (20), the error system from (7) can be written as

$$
\begin{aligned}
\dot{\tilde{e}}_{1_{i}}= & \tilde{A}_{11} \tilde{e}_{1_{i}}+\tilde{A}_{12} e_{2_{i}}+\left[I_{n-p}-L\right] G_{l_{i}} e_{y_{i}} \\
& -\alpha(t) \sum_{j=1}^{N} \mathcal{L}_{i j}\left(\tilde{\Gamma}_{11} \tilde{e}_{1_{j}}+\tilde{\Gamma}_{12} e_{2_{j}}\right) \\
\dot{e}_{2_{i}}= & A_{21} \tilde{e}_{1_{i}}+\tilde{A}_{22} e_{2_{i}}+D_{2} \xi_{i}(.)+G_{2_{i}} e_{y_{i}}+C_{2}^{\mathrm{T}} \nu_{e q, i} \\
& -\alpha(t) \sum_{j=1}^{N} \mathcal{L}_{i j}\left(\Gamma_{21} \tilde{e}_{1_{j}}+\tilde{\Gamma}_{22} e_{2_{j}}\right)
\end{aligned}
$$

for $i=1, \ldots, N$ where

$$
\left[\begin{array}{ll}
\tilde{A}_{11} & \tilde{A}_{12} \\
A_{21} & \tilde{A}_{22}
\end{array}\right]=T_{L} A T_{L}^{-1}
$$

and $\tilde{A}_{11}:=\left(A_{11}-L^{o} A_{211}\right)$. The local coupling matrix terms are obtained from the partition

$$
\left[\begin{array}{ll}
\tilde{\Gamma}_{11} & \tilde{\Gamma}_{12} \\
\Gamma_{21} & \tilde{\Gamma}_{22}
\end{array}\right]=T_{L} \Gamma T_{L}^{-1}
$$

where $\tilde{\Gamma}_{11} \in \mathbb{R}^{(n-p) \times(n-p)}$. 
Define

$$
G_{2_{i}}:=-\tilde{A}_{22} C_{2}^{\mathrm{T}}+\Psi C_{2}^{\mathrm{T}}
$$

and

$$
G_{1_{i}}:=L G_{2_{i}}-\tilde{A}_{12} C_{2}^{\mathrm{T}}
$$

for $i=1, \ldots, N$. In (25), $\Psi \in \mathbb{R}^{p \times p}$ is a designer selected stable matrix.

Let $\tilde{e}_{1}=\operatorname{Col}\left(\tilde{e}_{1_{1}}, \ldots \tilde{e}_{1_{N}}\right)$ and $e_{2}=\operatorname{Col}\left(e_{2_{1}}, \ldots e_{2_{N}}\right)$. Substituting $G_{1_{i}}$ and $G_{2_{i}}$ in (21)-(22), the error dynamics at a network level in the transformed coordinates can be written as

$$
\begin{aligned}
\dot{\tilde{e}}_{1}= & \left(\left(I_{N} \otimes \tilde{A}_{11}\right)-\alpha(t)\left(\mathcal{L} \otimes \tilde{\Gamma}_{11}\right)\right) \tilde{e}_{1}+\Phi_{1}\left(t, e_{2}\right) \\
\dot{e}_{2}= & \left(\left(I_{N} \otimes A_{21}\right)-\alpha(t)\left(\mathcal{L} \otimes \Gamma_{21}\right)\right) \tilde{e}_{1}+\Phi_{2}\left(t, e_{2}\right) \\
& +\left(I_{N} \otimes \Psi\right) e_{2}+\left(I_{N} \otimes D\right) \xi(x, u)+\left(I_{N} \otimes C_{2}^{\mathrm{T}}\right) \nu(28
\end{aligned}
$$

where $\nu:=\operatorname{Col}\left(\nu_{1}, \ldots \nu_{N}\right)$ and $\xi(x, u)=\mathcal{C o l}\left(\xi_{i}(\cdot), \ldots, \xi_{N}(\cdot)\right)$. In $(27)-(28), \Phi_{1}\left(t, e_{2}\right):=-\alpha(t)\left(\mathcal{L} \otimes \tilde{\Gamma}_{12}\right) e_{2}$ and $\Phi_{2}\left(t, e_{2}\right):=-\alpha(t)\left(\mathcal{L} \otimes \tilde{\Gamma}_{22}\right) e_{2}$.

Since $\Phi_{1}\left(t, e_{2}\right)$ is linear w.r.t. $e_{2}$

$$
\left\|\Phi_{1}\left(t, e_{2}\right)\right\| \leq \alpha(t) K_{1}\left\|e_{2}\right\|
$$

holds for all $\left(t, e_{2}\right) \in \mathbb{R} \times \mathbb{R}^{p N}$, where $K_{1}$ is a positive scalar.

Define $\mathcal{V}\left(\tilde{e}_{1}\right):=\tilde{e}_{1} \tilde{P} \tilde{e}_{1}$, where $\tilde{P}=\left(I_{N} \otimes P_{1}\right)$ and $P_{1}$ is a s.p.d matrix satisfying conditions (18)-(19). Further define $\tilde{\mathcal{V}}=\sqrt{\mathcal{V}}$. It follows that

$$
\left.\dot{\mathcal{V}}\right|_{(27)}=-\frac{\tilde{q}}{\lambda_{\max }\left(P_{1}\right)} \tilde{\mathcal{V}}^{2}+2 \mathcal{V} \sqrt{\lambda_{\max }\left(P_{1}\right)} \alpha(t) K_{1}\left\|e_{2}\right\|
$$

where $\tilde{q}$ is the positive scalar quantity in (19) and from Rayleigh's inequality (Khalil [1992])

$$
\lambda_{\min }\left(P_{1}\right)\left\|e_{1}\right\|^{2} \leq \mathcal{V}\left(\tilde{e}_{1}\right) \leq \lambda_{\max }\left(P_{1}\right)\left\|e_{1}\right\|^{2}
$$

Since by definition $\mathcal{V}=\tilde{\mathcal{V}}^{2}$ it follows $\dot{\mathcal{V}}=2 \dot{\tilde{\mathcal{V}}} \tilde{\mathcal{V}}$ and hence

$$
\dot{\tilde{\mathcal{V}}}\left(\tilde{e}_{1}\right) \leq-\frac{\tilde{q}}{2 \lambda_{\max }\left(P_{1}\right)} \tilde{\mathcal{V}}+\alpha(t) \sqrt{\lambda_{\max }\left(P_{1}\right)} K_{1}\left\|e_{2}\right\|
$$

which for notational simplicity can be written as

$$
\dot{\tilde{\mathcal{V}}} \leq-m_{0} \tilde{\mathcal{V}}+m_{1} \alpha(t)\left\|e_{2}(t)\right\|
$$

for appropriate positive scalars $m_{0}$ and $m_{1}$.

Define adaptive filter dynamics as

$$
\dot{r}_{1}(t)=-m_{0} r_{1}(t)+m_{1} \alpha(t)\left\|e_{2}(t)\right\|
$$

where $r_{1} \in \mathbb{R}$ is the adapting scalar element. Note that $e_{y_{i}}=C_{2} e_{2_{i}}$, where $C_{2}$ is orthogonal, which implies $\left\|e_{y_{i}}\right\|=$ $\left\|e_{2_{i}}\right\|$ and $\left\|e_{y}\right\|=\left\|e_{2}\right\|$. Hence (33) can be written as

$$
\dot{r}_{1}(t)=-m_{0} r_{1}(t)+m_{1} \alpha(t)\left\|e_{y}(t)\right\|
$$

From first principles, it can be seen that $\left\|r_{1}(t)\right\|>\tilde{\mathcal{V}}(t)$ for all time $t>0$, provided $r_{1}(0) \geq \tilde{\mathcal{V}}(0)$. Note that $r_{1}(t)$ is an adaptive gain which can be realized in finite time and depends only on $\left\|e_{y}\right\|$. Consequently if $r_{1}(0)>\tilde{\mathcal{V}}(0)$ then

and so

$$
r_{1}(t)^{2}>\mathcal{V}(t) \geq \lambda_{\min }\left(P_{1}\right)\left\|\tilde{e}_{1}\right\|^{2}
$$

$$
r_{1}(t) \geq \sqrt{\lambda_{\min }\left(P_{1}\right)}\left\|\tilde{e}_{1}(t)\right\|
$$

for all time. Now consider the dynamics in (28). Again it can be argued that

$$
\left\|\Phi_{2}\left(t, e_{2}\right)\right\| \leq \alpha(t) K_{2}\left\|e_{2}\right\|
$$

where $K_{2}$ is some positive constant. The objective is to show the reachability condition (Edwards and Spurgeon [1998], Utkin [1992]) can be satisfied to show that a sliding motion exists in finite time.

Define another adaptive scalar gain $r_{2}$ according to the following dynamics

$$
\dot{r}_{2}(t)=\gamma\left\|e_{y}(t)\right\|
$$

where $\gamma$ is a positive scalar. Also define

$$
e_{r}(t):=\left\|D_{2}\right\| \beta_{0}-r_{2}(t)
$$

In addition, assume that the matrix $\Psi$ in (25) has the form $\Psi:=\psi I_{p}$ where $\psi \in \mathbb{R}^{+}$is a design scalar which will be specified later. Consider

$$
\mathbf{V}=e_{2}^{\mathrm{T}} e_{2}+\frac{1}{\gamma} e_{r}^{2}
$$

Taking the derivative of $\mathbf{V}$ along the trajectory of (27) (28) yields

$$
\begin{aligned}
\dot{\mathbf{V}} \leq & \frac{2}{\gamma} e_{r} \dot{e}_{r}+2\left\|e_{2}\right\|\left\|\left(I_{N} \otimes A_{21}\right)\right\|\left\|\tilde{e}_{1}\right\|-2 \psi\left\|e_{2}\right\|^{2} \\
& +2 \alpha(t)\left\|\left(\mathcal{L} \otimes \Gamma_{21}\right)\right\|\left\|e_{2}\right\|\left\|\tilde{e}_{1}\right\|+2 \alpha(t)\left\|\left(\mathcal{L} \otimes \tilde{\Gamma}_{22}\right)\right\|\left\|e_{2}\right\|^{2} \\
& +2 K_{2}\left\|e_{2}\right\|^{2}+2\left\|e_{2}\right\|\left\|D_{2}\right\| \beta_{0}-2 e_{2}^{\mathrm{T}}\left(I_{N} \otimes C_{2}^{\mathrm{T}}\right) \nu
\end{aligned}
$$

It is easy to see that

$$
e_{2}^{\mathrm{T}}\left(I_{N} \otimes C_{2}^{\mathrm{T}}\right)=e_{2}^{\mathrm{T}}\left(I_{N} \otimes C_{2}\right)^{\mathrm{T}}=\left(\left(I_{N} \otimes C_{2}\right) e_{2}\right)^{\mathrm{T}}=e_{y}^{\mathrm{T}}
$$

Applying the definition of $\nu$ in (6), it follows:

$$
e_{2}^{\mathrm{T}}\left(I_{N} \otimes C_{2}^{\mathrm{T}}\right) \nu=-\rho \sum_{i=1}^{N}\left\|e_{y i}\right\| \leq-\rho\left\|e_{y}\right\|
$$

Consequently, if $\psi>\left(\alpha(t)\left\|\left(\mathcal{L} \otimes \tilde{\Gamma}_{22}\right)\right\|+K_{2}\right)$ then

$$
\begin{aligned}
\dot{\mathbf{V}} \leq & 2\left\|e_{2}\right\|\left\|\left(I_{N} \otimes A_{21}\right)\right\|\left\|\tilde{e}_{1}\right\|+2 \alpha(t)\left\|\left(\mathcal{L} \otimes \Gamma_{21}\right)\right\|\left\|e_{2}\right\|\left\|\tilde{e}_{1}\right\| \\
& +2\left\|e_{2}\right\|\left\|D_{2}\right\| \beta_{0}-2 \rho\left\|e_{y}\right\|+\frac{2}{\gamma} e_{r} \dot{e}_{r}
\end{aligned}
$$

Let $a_{21}=\left\|I_{N} \otimes A_{21}\right\|=\left\|A_{21}\right\|$ and $\gamma_{21}=\alpha(t)\left\|\mathcal{L} \otimes \Gamma_{21}\right\|$. Since $\alpha(t)$ is bounded by $\hat{\alpha}$ then such a constant exists. Moreover, note that $r_{1}(t)>\sqrt{\lambda_{\min }\left(P_{1}\right)}\left\|\tilde{e}_{1}(t)\right\|$ for all $t>0$. Using these facts and substituting in (39), and since $\left\|e_{y}\right\|=\left\|e_{2}\right\|$, yields

$$
\dot{\mathbf{V}}=2\left(\frac{a_{21}+\gamma_{21}}{\sqrt{\lambda_{\min }\left(P_{1}\right)}} r_{1}(t)+\left\|D_{2}\right\| \beta_{0}-\rho(t)\right)\left\|e_{2}\right\|+\frac{2}{\gamma} e_{r} \dot{e}_{r}
$$

Define

$$
\rho(t):=\frac{\left(a_{21}+\gamma_{21}\right)}{\sqrt{\lambda_{\min }\left(P_{1}\right)}} r_{1}(t)+r_{2}(t)+r_{0}
$$

where $r_{0}$ is a positive design scalar. Subsequently, substituting the term $\rho(t)$ in (40) yields

$$
\begin{aligned}
\dot{\mathbf{V}} & \leq 2\left\|D_{2}\right\| \beta_{0}\left\|e_{2}\right\|-2 r_{2}(t)\left\|e_{2}\right\|-2 r_{0}\left\|e_{2}\right\|+\frac{2}{\gamma} e_{r} \dot{e}_{r} \\
& =2\left\|e_{2}\right\|\left(\left\|D_{2}\right\| \beta_{0}-r_{2}(t)\right)-2 r_{0}\left\|e_{2}\right\|+\frac{2}{\gamma} e_{r} \dot{e}_{r}
\end{aligned}
$$

Using the definition of $e_{r}$ in (37), the Lyapunov derivative in (42) is written as

$$
\dot{\mathbf{V}}=2 e_{r}\left\|e_{2}\right\|-2 r_{0}\left\|e_{2}\right\|+\frac{2}{\gamma} e_{r} \dot{e}_{r}
$$

It follows from (37) that by definition $\dot{e}_{r}=-\dot{r}_{2}$ and substituting for $\dot{r}_{2}$ from (36) yields 


$$
\dot{\mathbf{V}} \leq-2 r_{0}\left\|e_{2}\right\|
$$

The stability argument is thus complete.

The exposition above demonstrates that the signals $e_{1}$ $e_{2}$ and $e_{r}$ remain bounded. However, if over an interval of time $\left[\begin{array}{ll}t_{1} & t_{2}\end{array}\right]$ the gain $r_{2}(t)$ satisfies $r_{2}(t)>\left\|D_{2} \xi(t)\right\|$ then sliding will occur within $t_{s}=\left\|e_{2}\left(t_{1}\right)\right\| / r_{0}$ units of time provided $t_{1}+t_{s} \leq t_{2}$. This follows from employing the Lyapunov function $V_{s}=e_{2}^{\mathrm{T}} e_{2}$ and noting that, using arguments similar to those leading up to (42),

$$
\begin{aligned}
\dot{V}_{s} & \leq 2\left\|D_{2}\right\|\|\xi\|\left\|e_{2}\right\|-2 r_{2}(t)\left\|e_{2}\right\|-2 r_{0}\left\|e_{2}\right\| \\
& \leq-2 r_{0}\left\|e_{2}\right\|
\end{aligned}
$$

if $r_{2}(t)>\left\|D_{2} \xi(t)\right\|$. Consequently

$$
\dot{V}_{s} \leq-2 r_{0} \sqrt{V_{s}}
$$

and $\left\|e_{2}\right\|=0$ in a time $t_{s} \leq\left\|e_{2}\left(t_{1}\right)\right\| / r_{0}$. Once sliding is achieved, $e_{2}=0$ and from (36) the gain $r_{2}(t)$ no longer increases, and sliding will be maintained provided the gain $r_{2}\left(t_{1}+t_{s}\right)>\left\|D_{2} \xi(t)\right\|$ for all $t>t_{1}+t_{s}$. If this does not hold (which is possible if $\left.r_{2}\left(t_{1}+t_{s}\right)<\left\|D_{2}\right\| \beta_{0}\right)$ then sliding may be temporarily broken, and from $(36)$, the gain $r_{2}(t)$ will increase and sliding will be regained in finite time using arguments similar to those above. If at any point $r_{2}(t)>\left\|D_{2}\right\| \beta_{0}$, sliding will be achieved and maintained for all subsequent time.

\section{NUMERICAL EXAMPLE}

To demonstrate the theory developed in this paper, a network of six Chua oscillators is considered. The following Lur'e type (Khalil [1992]) representation for Chua's circuit is adopted:

$$
\begin{aligned}
& \dot{x}_{i}=A x_{i}+D \xi_{i}\left(x_{i}\right)-\alpha(t) \sum_{j=1}^{N} \mathcal{L}_{i j} \Gamma x_{j}+B u_{i} \\
& y_{i}=C x_{i}
\end{aligned}
$$

where

$$
A=\left[\begin{array}{ccc}
-a l_{1} & a & 0 \\
1 & -1 & 1 \\
0 & -b & 0
\end{array}\right], D=\left[\begin{array}{c}
-a\left(l_{0}-l_{1}\right) \\
0 \\
0
\end{array}\right], C=\left[\begin{array}{lll}
1 & 0 & 0 \\
0 & 1 & 0
\end{array}\right]
$$

The nonlinearity is $\xi_{i}\left(x_{i}\right)=\frac{1}{2}\left(\left|x_{i 1}+c\right|-\left|x_{i 1}-c\right|\right)$, which has a sector bound $[0,1]$. The chosen values of the parameters are $a=9, b=14.286, c=1, l_{0}=$ $-1 / 7, l_{1}=2 / 7$ in order to obtain the double scroll attractor(Wu [2007], Suykens et al. [1999]). The underlying graph topology of Chua oscillators, which are coupled via resistors, is based on the nearest neighbour rule as in Mesbahi and Egerstedt [2010]. The interconnection matrix $\Gamma=\mathcal{D} \operatorname{iag}(1,0,0)$ which models the interconnection among the states of the Chua oscillators. The time varying coupling $\alpha(t)$ is modelled as a sinusoidal signal with input frequency of $0.5 \mathrm{~Hz}$ and amplitude 0.5 and bias 1.0 and therefore $\alpha(t)$ assumed to have a known upper bound $\hat{\alpha}=2$.

As in Suykens et al. [1999], the control input matrix $B$ is taken as the identity. The control strategy adopted in the supervisory level node is the static state error feedback controller discussed in Suykens et al. [1999]. Synchronisation to a pre-defined master system behaviour (whose initial conditions are $\left.x_{\text {master }}(0)=[0.0196,0.6813,0.3795]\right)$ is obtained with full static-state error feedback to the individual nodes using the control signal $u_{i} \in \mathbb{R}^{m}$ with

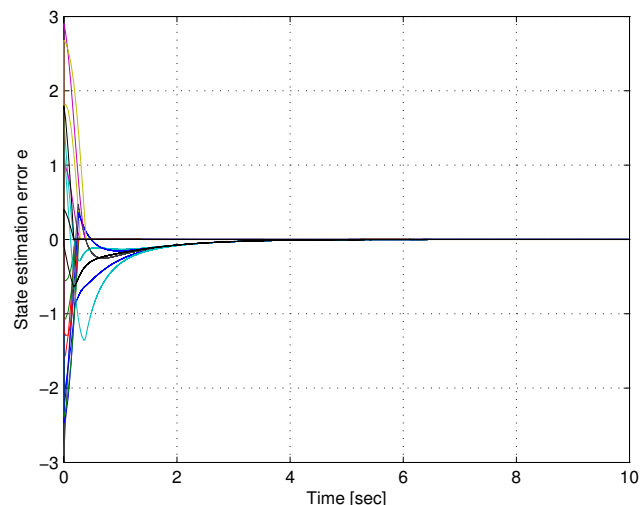

Fig. 1. State estimation error
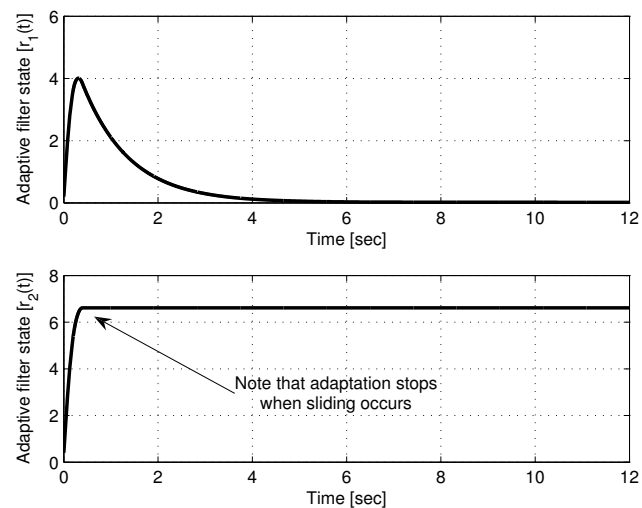

Fig. 2. The states of the adaptive filter dynamics $\left(r_{1}, r_{2}\right)$

the feedback matrix $F \in \mathbb{R}^{m \times n}$ with the intention of obtaining double-scroll attractor behaviour, and to force all the individual nodes, which are randomly initialized, to reach consensus to this trajectory. All the nodes of the network are brought to synchronisation.

The main objective is robust reconstruction of all the states of all the nodes of the network. The 4-block partition structure in (3) is obtained by suitable coordinate transformation The triple $(A, D, C)$ satisfies the Assumptions (1) - (3). The network system is then implemented in this coordinate structure for simplicity. At a centralised level, the states of every node level system of the network are estimated from the measurement signals $y_{i}$ using the proposed adaptive sliding mode observer in (5). Here, one assumption is that each node communicates its output information to the supervisory monitoring node in order to compute $\left\|e_{y}\right\|$ centrally, since this information is used in the adaptive filter dynamics in (34) and (36) of the proposed sliding mode observer. For implementation (36), is replaced with an adaptation law involving a 'dead-zone' so that $\dot{r}_{2}(t)=0$ if $\left\|e_{y}\right\| \leq \epsilon$ otherwise $\dot{r}_{2}(t)=\gamma\left\|e_{y}\right\|$ where $\epsilon$ is a small positive scalar. In the simulations which follow $\epsilon=0.0001$. The design freedom $\tilde{q} \in \mathbb{R}^{+}$in (19) is chosen as unity.

Solving the inequalities in (18)-(19), a Lyapunov matrix, $P_{1}$ (in the present case, a scalar variable) is synthesized. Following the norm bounds discussed in subsection 3.2, appropriate gains for the adaptive law in (34) and (36) have been selected as $m_{0}=-1, m_{1}=3.0425$ and $\gamma=5$. In (41), the bounds and the design scalars are selected as $a_{21}=1, \gamma_{21}=0, K_{1}=4.3028, K_{2}=0$ and $r_{0}=2$. 

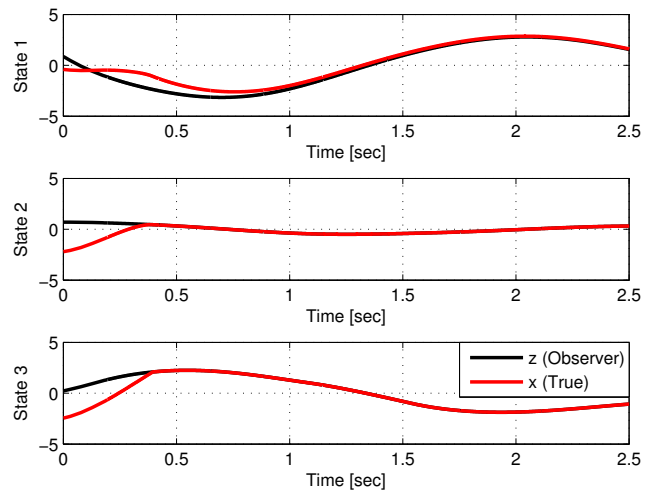

Fig. 3. State estimation of one individual node

The simulations are carried out in Matlab 7.3.0.267 (R2006b) and the Simulink version 6.5 environment. The solver used is ode15s / stiff with a maximum and minimum step size of 1e-3 and 1e-6 respectively. Figure 1 shows the entire state estimation error $e=\mathcal{C} o l\left(e_{1}, \ldots, e_{6}\right)$. Figure 2 shows the states $r_{1}(t)$ and $r_{2}(t)$ of the adaptive filter dynamics defined in (34) and (36) (shown only for a short span of time). In Figure 2, notice that the adaptation of the gains converges at the instance of the occurrence of the sliding motion which can also be observed in 1 . To demonstrate the efficacy of the proposed state reconstruction, an individual node is selected randomly and the true states and the estimated states are shown in Figure 3.

\section{CONCLUSION}

This paper has focused on reconstructing robustly the entire state information in a complex network dynamical system at a supervisory node level. Novel adaptive sliding mode observers are designed for this purpose. The proposed observers robustly reconstruct the states in the presence of unknown bounded coupling strengths and a class of unknown bounded uncertainties/nonlinearities/disturbances. The stability conditions derived for the network observer depend only on the individual nodes of the network. The design procedure is formulated as an LMI optimisation problem performed at a node level. A network of Chua circuits with six nodes is used to demonstrate the novelty of the proposed approach.

\section{REFERENCES}

Azemi, A. and Yaz, E. (2000). Sliding mode adaptive observer approach to chaotic synchronization. Journal of Dynamic Systems, Measurement, and Control, 122, $758-765$

Bejarano, F., Fridman, L., and Poznyak, A. (2007). Hierarchical observer for strongly detectable systems via second order sliding mode. In Proceedings of the IEEE Conference on Decision and Control, 3709-3713.

Chen, W. and Saif, M. (2007). Actuator fault diagnosis for uncertain linear systems using a high-order sliding-mode robust differentiator. International Journal of Robust and Nonlinear Control, 18.

Drakunov, S. and Utkin, V. (1995). Sliding mode observers: tutorial. In Proceedings of the 34th IEEE Conference of Decision and Control, 3376-3378.

Edwards, C. and Spurgeon, S. (1994). On the development of discontinuous observers. International Journal of Control, 59, 1211-1229.

Edwards, C. and Spurgeon, S. (1998). Sliding Mode Control: Theory and Applications. Taylor \& Francis.

Edwards, C., Spurgeon, S., and Patton, R. (2000). Sliding mode observers for fault detection. Automatica, 36, 541553 .
Fax, J.A. and Murray, R.M. (2004). Information flow and cooperative control of vehicle formations. IEEE Trans. Aut. Control, 49, 1465-1476.

Floquet, T. and Barbot, J. (2004). A sliding mode approach for unknown input observers for linear systems. In Proceedings the IEEE Conference on Decision and Control, Bahamas.

Floquet, T. and Barbot, J. (2006). An observability form for linear systems with unknown inputs. International Journal of Control, 79, 132-139.

Fridman, L., Davila, J., and Levant, A. (2007a). Highorder sliding-mode observation and fault detection. Proceedings of the IEEE Conference on Decision and Control, 4317-4322.

Fridman, L., Shtessel, Y., Edwards, C., and Yan, Y. (2007b). Higher order sliding mode observer for state estimation and input reconstruction in nonlinear systems. International Journal of Robust and Nonlinear Control, 18, 399-412.

$\mathrm{Ji}, \mathrm{X}$. and Chen, G. (2007). On the v-stability of complex dynamical networks. Automatica, 43, 1049-1057.

Kambhampati, C., Perkgoz, C., Patton, R.J., and Ahamed, W. (2007). An interconnection predictive approach to fault-tolerant control in network control systems. Journal of Systems and Control Engineering, $221,885-894$.

Khalil, H. (1992). Nonlinear Systems. Prentice Hall, Englewood Cliffs NJ.

Koshkouei, A. and Zinober, A. (2000). Sliding mode controller-observer design for multivariable linear systems with unmatched uncertainty. Kybernetika, 36, 95115.

Mariottini, G.L., Pappas, G., Prattichizzo, D., and Daniilidis, K. (2005). Vision-based localization of leaderfollower formations. Proceedings of Conference on Decision and Control and European Control Conference.

Menon, P. and Edwards, C. (2008). Rank constrained stabilization of complex networks. In Proceedings of the American Control Conference, Seattle.

Mesbahi, M. and Egerstedt, M. (2010). Graph Theoretic Methods in Multiagent Networks. Princeton Series in Applied Mathematics.

Noijen, S., Lambrechts, P., and Nijmeijer, H. (2005). An observer-controller combination for a unicycle mobile robot. International Journal of Control, 78, 81-87.

Patton, R.J., Kambhampati, C., Casavola, A., Zhang, P., Ding, S., and Sauter, A.D. (2007). Generic strategy for fault-tolerance in control systems distributed over a network. European Journal of Control, 13, 280-296.

Raoufi, R. and Zinober, A. (2006). Adaptive sliding mode observers in uncertain chaotic cryptosystems with a relaxed matching condition. International Workshop on Variable Structure Systems, 220-225.

Royle, G. and Godsil, C. (2001). Algebraic graph theory. Springer Verlag, New York.

Suykens, J.A.K., Curran, P.F., and Chua, L.O. (1999). Robust synthesis for master slave synchronization of lure systems. IEEE Transactions on circuits and systems-I: Fundamental theory and applications, 46, 841-850.

Utkin, V. (1992). Sliding Modes in Control Optimization. Springer-Verlag, Berlin.

Wu, C.W. (2007). Synchronisation of complex networks of nonlinear dynamical systems. World scientific publishing company.

Wu, Q. and Saif, M. (2007). Robust fault detection and diagnosis for a multiple satellite formation flying system using second order sliding mode and wavelet networks. Proceedings of American Control Conference.

Yan, J.J., Hung, M., Chiang, T.Y., and Yang, Y. (2006). Robust synchronization of chaotic systems via adaptive sliding mode control. Physica A, 356, 220-225. 